\title{
Publisher's Note: Haldane-Hubbard Mott insulator: From Tetrahedral Spin Crystal to Chiral Spin Liquid [Phys. Rev. Lett. 116, 137202 (2016)]
}

Ciarán Hickey, Lukasz Cincio, Zlatko Papić, and Arun Paramekanti

(Received 26 April 2016; published 2 May 2016)

DOI: 10.1103/PhysRevLett.116.189901

This paper was published online on 1 April 2016 with an error in the Acknowledgments. On page 5, the first sentence of the Acknowledgments should read as "We thank R. Desbuquois, K. Hwang, G. Jotzu, S. Sachdev, D. N. Sheng, A. Thomson, and S. Whitsitt for useful discussions." The Acknowledgments have been corrected as of 4 April 2016. The Acknowledgments are correct in the printed version of the journal. 